\title{
The current status of studies of human exposure assessment of microplastics and their health effects: a rapid systematic review
}

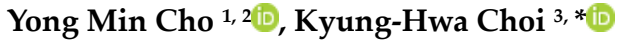 \\ ${ }^{1}$ Environmental Health Center, Seokyeong University, Seoul, Republic of Korea \\ ${ }^{2}$ Department of Nano Chemical and Biological Engineering, Seokyeong University, Seoul, Republic of Korea \\ ${ }^{3}$ Department of Preventive Medicine, Dankook University College of Medicine, Cheonan, Chungnam, Republic of Korea
}

*Correspondence: rosach72@hanmail.net

Received: October 17, 2020 Accepted: January 11, 2021

\begin{abstract}
This rapid systematic review aims to summarize studies of human exposure to microplastics (MPs) and their health effects. For the systematic literature review, we separately searched for review articles and original articles published from 2000 to 2019 in the PubMed, NDSL, KMBASE, and Cochrane databases. A total of 276 review articles and 475 original articles were independently evaluated and eligibility of each article was assessed by two researchers. Finally, 8 review articles and 13 original articles were selected for analysis. There was no standardized methodology for determining human exposure to MPs. Therefore, the size, shape, color, and chemical composition of MPs were considered as factors that affected human exposure to MP. We ascertained that human exposure to MPs occurs through two major routes: inhalation and ingestion. The general population can be exposed to MPs through the food chain, food, and mineral water. Thus, there are multiple scenarios for the exposure process. The only exposure marker in humans is the detection and measurement of MPs in feces. Health effects of human exposure to MPs include respiratory effects from inhalation, digestive effects from ingestion, oxidative stress, and cancer. However, there are few studies of the effects of MP exposure in the general population. Based on a systematic review, we propose a standardized methodology to identify various exposure scenarios to facilitate studies of human exposure to MPs and their health effects.
\end{abstract}

Keywords: microplastics, rapid systematic review, human exposure

\section{Introduction}

The term microplastics (MPs) was initially coined by Thomson et al. and MPs were defined as very small plastic particulates and fibers with a particle size less than $5 \mathrm{~mm} \mathrm{[1,2].} \mathrm{MPs} \mathrm{are} \mathrm{classified} \mathrm{into} \mathrm{primary} \mathrm{MPs,} \mathrm{which} \mathrm{are} \mathrm{produced}$ for special applications, such as cosmetics, toothpastes, and detergent products, and secondary MPs, which are derived from fragments of plastic discarded in the sea and on land [3]. Small MPs, with particle size between 1 and $100 \mathrm{~nm}$, are called nanoplastics [4]. With the global increase in plastic consumption over the past decades, MPs have become widely distributed in the oceans worldwide and their proportion in the environment is steadily increasing [5]. MPs are detected in most environmental media, such as oceans, soils, lakes, and sediments, and can be referred to as "microplastic pollution" $[4,6]$. Environmental MP pollution can have a negative effect on organisms and ecosystems in the environment and can even affect human health [6]. Several studies have raised concerns about the health effects of MPs [7-9]. MPs that are present in various environmental media, including drinking water and manmade products, can directly enter the human body through ingestion, inhalation, and skin contact [7]. In addition, the transition of MPs to the final predator through the food chain in an ecosystem contaminated with MPs as well as the human consumption of these final predators constitute important models of human exposure to MPs [8].

With regard to the determination of human toxicity as an environmental hazard, MPs are similar to particulate matter (PM) in that they are complex components, and their hazard effect on the human body may be determined by the chemical composition and physical form like size of PMs. However, exposure to PMs is mainly through inhalation whereas MP exposure occurs in more diverse scenarios, with both inhalation and ingestion being important routes of exposure [7].

A few researchers envisaged that MPs in environmental media, food, and consumer products are disseminated to the general population through various channels and can cause health effects [7,9]. Currently, environmental health and toxicity scientists are faced with the challenge of establishing a hypothesis for the exposure assessment and health effect assessment of MPs in human populations, writing exposure scenarios, and establishing research protocols. However, such 
exposure models have only been suggested in the academic world, and few studies have evaluated or quantified the actual human exposure of MPs so far. Moreover, few studies have been conducted to analyze the possible human exposure to MPs and the cause and effect of MP exposure on human health.

Therefore, this rapid systematic review aims to establish the possibilities of human exposure and the effects of MPs by reviewing the literature to develop a standard methodology whereby studies can assess human exposure to MPs and the health effects of MPs.

\section{Materials and methods}

The research question in this study consists of the PECO statement has already been introduced in the other environmental health study [10]. The PECO statement for the study; P (Population: general population), E (Exposure: exposure to all types of MPs), C (Comparator: object or group not exposed to MPs), and O (Outcome: MPs exposure and relationship - all types of health effects) was established.

\section{Search strategy and data source}

A rapid systematic review was performed in two stages. First, we searched for systematic review or literature review articles that reported the latest information and insights on human exposure assessment or health effect assessment of MPs (Figure 1). The second step was to search for original research articles. PubMed was the primary database for the searches; however, we searched the NDSL (National Digital Science Library), KMBASE (Korean Medical Database), and Cochrane Library databases. The search was carried out in May 2020. The MeSH terms "microplastic" and "micro plastic" were used as search terms, and a combination of the search terms "microplastic" [MeSH Terms] OR "micro plastic" OR "plastic debris" AND "human exposure" OR "human health" OR "health" [MeSH Terms] were used with all searches by [All fields] condition. The entire process of searching and reviewing the literature was carried out according to the guidelines of Preferred Reporting Items for Systematic Reviews and Meta-Analysis (PRISMA) [11], and two researchers , the authors, independently reviewed the search results.

\section{Study inclusion and exclusion}

Articles that were in languages other than English including Korean were excluded from the review as were studies of the ecosystems or environmental media. Animal studies were excluded, although studies that suggested the possibility of human exposure through the food chain and studies that showed the potential for health effects by extrapolating the results of animal experiments to the human body were included in the review. The search, selection, and review of the articles were conducted by two independent researchers ; disagreements, if any, on the inclusion of any article in the review were resolved by the inclusion of that article in the review. No restrictions were imposed with regard to study subjects, types of exposure, comparative groups or confounding variables, and type of health effects.

As this review was not intended to be a meta-analysis that collects quantitative data, this review identified qualitative information on the exposure factors and pathways of MPs evaluated in each study, measurement methods, and the proposed health effects. The possibility of health effects was suggested based on the viewpoint from which the research was conducted and was not actually evaluated or measured in the studies.

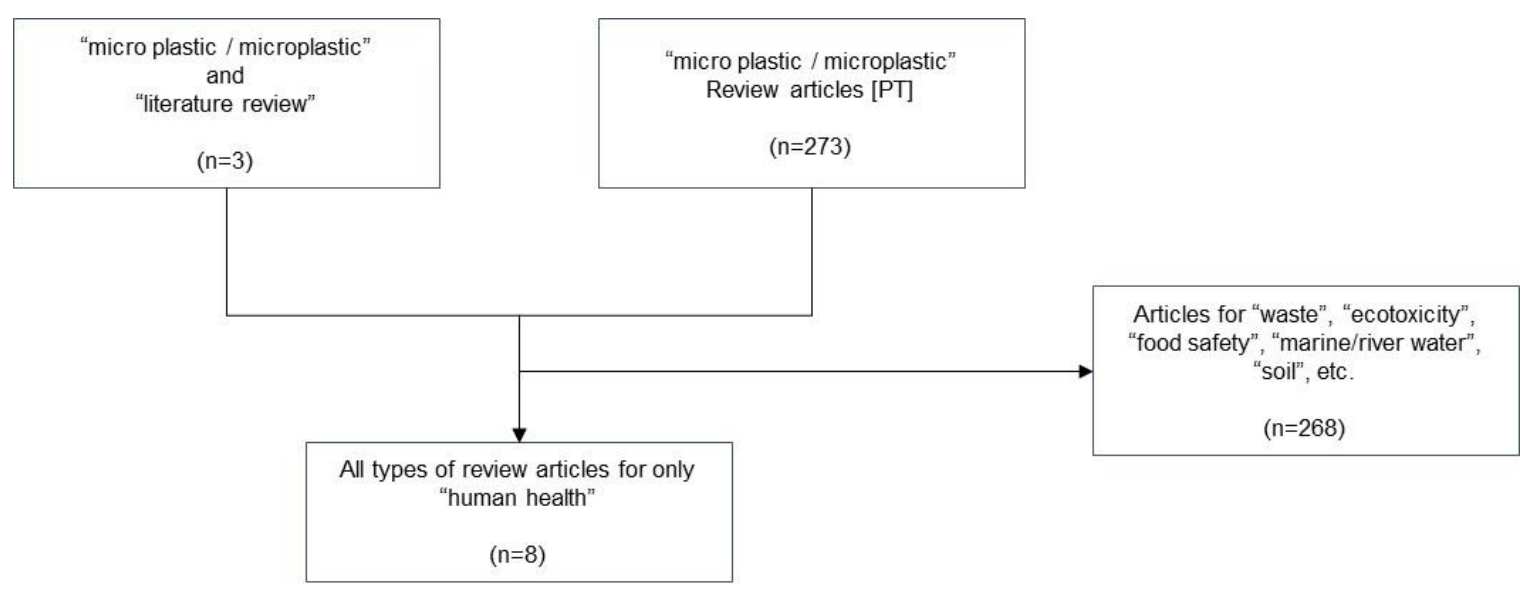

Figure 1 Flowchart of the identification of eligible studies among review articles 


\section{Quality assessment}

The risk-of-bias assessment of individual studies was performed using RoBANS-2.0, which is a risk-of-bias assessment tool for non-randomized studies [12]. RoBANS-2.0 constitutes eight items that are evaluated for comparability, selection of participants, confounding, measurement, blinding, assessment of outcomes, outcome data, and reporting bias, and each item is classified into one of three strata (designated by the indicated symbols) as follows: "low risk: +", "high risk: -“, and "unclear risk: ?".

\section{Results and discussion}

\section{Selection of review articles}

A total of 276 review articles were identified on the initial search, and 268 articles were excluded based on the exclusion criterion. Finally, eight review articles were selected for analysis in this review (Figure 1).

\section{Summary of review articles}

Table 1 shows the PECO components of eight review articles on MPs and human health that describe how the human body is exposed to MPs and expounds on the potential for human health effects (Table 1). MPs are usually defined as plastic particles measuring less than $5 \mathrm{~mm}$, but they can contain fiber particles $[7,13]$ and are classified according to size $[8,9]$. The route of MP exposure in the human body, as suggested by these studies, was indicated that the main exposure scenario was through the "food chain" [7-9, 13-16], and the consumption of seafood, such as fish and shellfish, was suggested as an important source of exposure. In addition, MPs were measured in foods and media, such as sea salt, beer, bottled water, and tap water [13-15, 17]. Exposure to air and occupational exposure scenarios were also suggested [7, 13, 15]. From the perspective of human health effects, scenarios wherein primary MPs are present in environmental media and are inhaled or ingested, as well as the pathways through which secondary MPs are transmitted to humans, considered the final predator, were investigated.

Most of the measurements of MP content in these exposed media have been performed through optical measurement methods. However, there were a few cases of exposure evaluation of MPs that were introduced into the human body, and although some studies have reported a quantitative evaluation of MPs in human fecal samples [15], it would be difficult to definitively aver that a standardized human exposure biomarker of MPs has been suggested. In addition, rather than quantitatively assessing the health effects of MPs, only some possibilities were raised through descriptive considerations in the eight articles included in this review.

Table 1 PECO (population, exposure, comparator, and outcome) components for the identification of review articles for analysis of human exposure to microplastics (MPs)

\begin{tabular}{|c|c|c|}
\hline & & Contents \\
\hline \multicolumn{2}{|l|}{ Population } & $\begin{array}{l}\text { - No limited certain population } \\
\text { - Workers exposed to microfibers [7] }\end{array}$ \\
\hline \multirow{3}{*}{ Exposure } & $\begin{array}{l}\text { Definition of } \\
\text { pollutant }\end{array}$ & $\begin{array}{l}\text { - General definition of MPs }{ }^{1)} \\
\text { - Microfiber }[13] \\
\text { - According to size: macro, micro, and nano }[8,9] \\
\text { - According to chemical components: phthalates, BPA, styrene, PAHs, microbes, POPs, } \\
\text { metals, andorganotin compounds }[8,14]\end{array}$ \\
\hline & Pathway/Media & $\begin{array}{l}\text { Food chain: seafood; shellfish }[7-9,13-16] \\
\text { - Food except for seafood: sea salt, beer, honey, sugar, can food, mineral water }[13-15,17] \text {, } \\
\text { and tap water [14] } \\
\text { - Exposure through air (inhalation) }[7,13,15]\end{array}$ \\
\hline & $\begin{array}{l}\text { Exposure } \\
\text { assessment }\end{array}$ & $\begin{array}{l}\text { - Mainly detects environmental media or food: FT-IR, Raman spectrometry, SEM/TEM, GC- } \\
\text { MS }[8,13,16] \\
\text { - Exposure assessment of humans: Human feces [15] }\end{array}$ \\
\hline \multicolumn{2}{|c|}{ Comparator } & - Not mentioned \\
\hline Outcome & & $\begin{array}{l}\text { - Digestive and immune effect }[8,15] \\
\text { - Lung effect by inhalation [13] } \\
\text { - Cancer, liver, and hormonal effect (toxicity effect of phthalate and BPA) [13] } \\
\text { - Oxidative stress, central nervous system, digestive, and immune system effect [9] } \\
\text { - Endocrine system and cancer [14] } \\
\text { - Occupational asthma (exposure to microfibers in the textile industry) [7] }\end{array}$ \\
\hline
\end{tabular}

1) Small plastic pieces $<5 \mathrm{~mm}$

PAHs: polycyclic aromatic hydrocarbons; BPA: bisphenol A; POPS: persistent organic pollutants; FT-IR: Fourier-transform infrared spectroscopy; SEM: scanning electron microscope; TEM: transmission electron microscope; GC-MS: gas chromatography - mass spectrometry 
Some studies have speculated that the health effects of MPs may be related to the chemical composition of MPs, such as polymers, phthalates, bisphenols, polycyclic aromatic hydrocarbons, persistent organic pollutants, and metals [8, 14]. The health effects of MPs are related to the toxicity of adsorbed chemicals, and the possibility of digestive effects from ingestion of MPs and lung effects from inhalation; cancer, oxidative damage, immune system effects, and hormonal effects have also been estimated. However, to date, it has not been identified in epidemiological studies.

\section{Selection of research articles}

A total of 475 original articles were identified on the initial search, and 462 articles were excluded due to duplication $(n=161)$, incompatibility $(n=270)$, and not reporting original research $(n=31)$. Thirteen original articles were selected for inclusion in this review (Figure 2).

\section{Summary of original articles}

After the initial search and a full-text review, 13 original research articles were finally selected (Figure 2). The definitions of MPs as hazardous substances were reported in these articles were plotted based on the following aspects: chemical species that can be considered, quantitative measurement methods and exposure factors, exposure routes, exposure assessment markers, early effects from exposure, and proposed health effects (Figure 3). Several studies have determined the types of MPs in plastic particles or nano-sized plastic particles [18], polymer particles, and micro-rubber [19]. The definition of a chemical substance as a polymer, such as polystyrene, polyethylene, polypropylene, polyvinyl chloride, and alkyd resin, and the standard reference materials of these substances were applied to analyze studies in the environmental media. In the quantitative evaluation of seafood and industrial products, the number of MPs per unit volume was mainly measured through optical instrumented analysis; however, in addition to the quantitative evaluation of the concept of concentration, an evaluation of the size, shape, and color of MPs was conducted. Thus, in the process of MP exposure to the human body, factors such as the absolute concentration (amount or number) of MPs as well as its shape and size can act as risk factors. These characteristics are likely to be factors that determine the mode of absorption of MPs by the human body, the amount of exposure, target organs, and resultant toxicity.

The environmental hazards of MPs can be considered similar to that of PMs in that there is no chemical definition. However, unlike PMs in which exposure is mainly inhalational, a study of the exposure routes of MPs must consider both inhalational exposure mechanisms [19-21] and ingestion-related exposure scenarios [22-26]. The possibility of exposure through ingestion was mainly considered and evaluated based on the intake of predators above the marine ecosystem in the food chain as well as the intake of mineral water or drinking water containing MPs. On the other hand, in cases where human feces were applied as a biomarker for evaluating the amount of human exposure through ingestion, an optical quantification method was used to assess the involuntary ingestion of MPs [22]. Ingestion is considered as the main exposure pathway of MPs and MPs are defined in their physical form. Therefore, the only methodology to measure MPs exposed to humans to date is the detection of MP itself in biospecimen excreted from the digestive tract. The method is different from general biomarkers of environmental chemical pollutants, which measuring levels of chemicals in blood or urine samples.

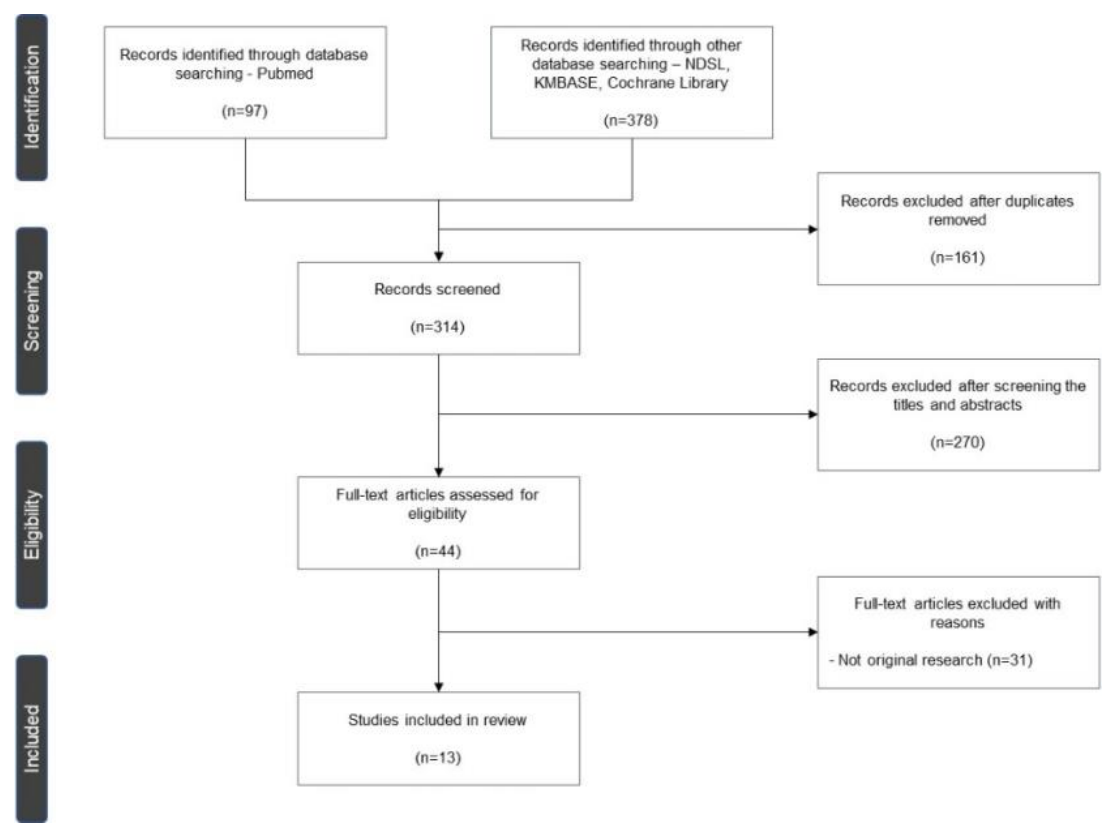

Figure 2 Flowchart of the identification for eligible studies from a search among original articles 
Cho and Choi I Human exposure to microplastics and their health effects

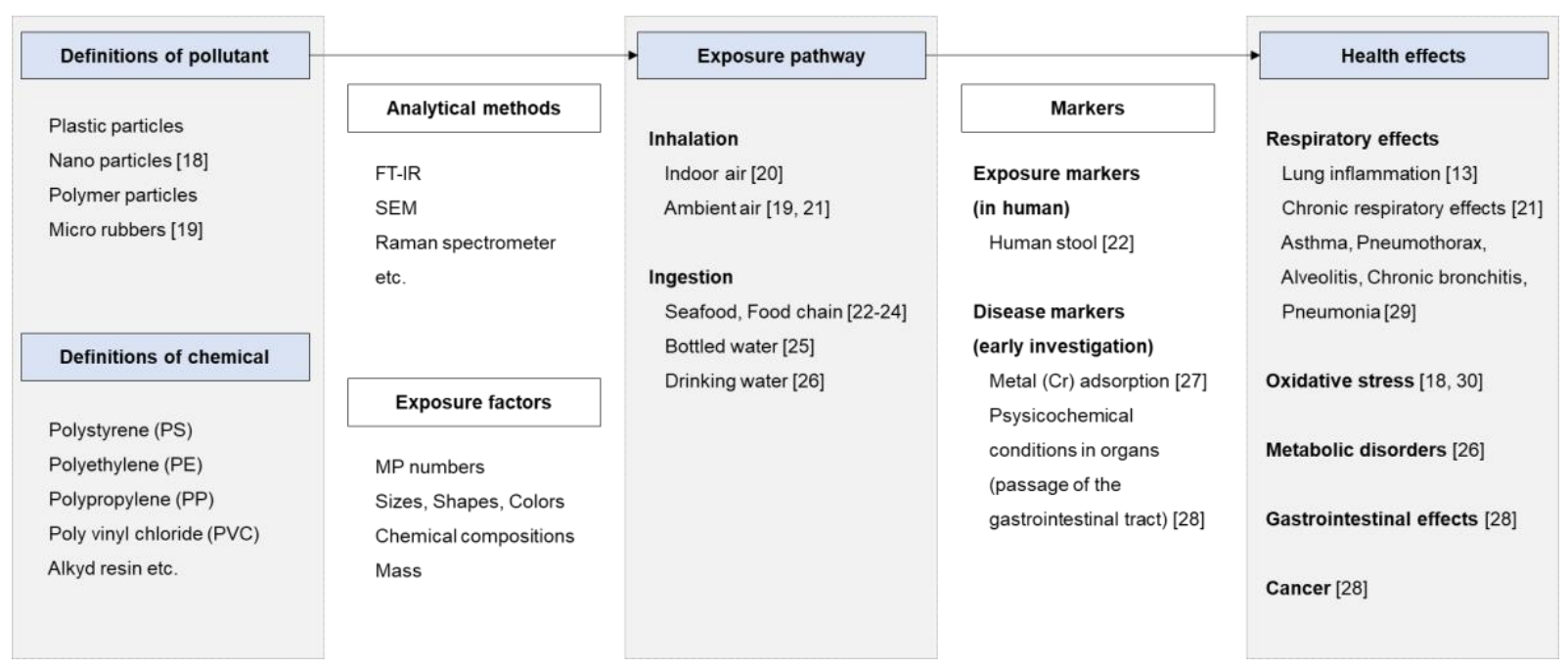

Figure 3 Summary of the systematic review - human exposure to microplastics (MPs) and human health effects.

FT-IR: Fourier-transform infrared spectroscopy; SEM: scanning electron microscopy.

The early effects of MPs on the body have been suggested to be mediated by the absorption or alteration of the digestive system [27, 28], which is mainly based on ingestion exposure scenarios. Meanwhile, the possibility of respiratory exposure, such as lung inflammation [13], chronic respiratory effects [21], asthma, pneumothorax, alveolitis, chronic bronchitis, and pneumonia [29], were suggested. In addition, it has been suggested that the influx of MPs into the body may induce oxidative stress [18, 30], metabolic disorders [26], gastrointestinal effects [28], and cancer [28] in the long term, although the actual health impact assessment has not been sufficiently conducted in these studies.

\section{Overview}

The results of this review show the current stage of research on the effects of MPs on human health. In other words, reports on human exposure and effects of MPs are at a nascent stage, and observations of various phenomena are listed instead of the creation of more original research evidence. There is relatively much evidence to indicate that MPs have contaminated environmental media, although it is difficult to obtain evidence that this environmental MP contamination can cause human exposure and health effects. Observational studies on the human body or population have not been reported to date, and the possibility of human exposure and health effects have been suggested indirectly through the measurement of the MPs content of ecosystems, foods, and household goods. There is a lack of clear evidence of human exposure and health effects; nonetheless, the hypothesis that the general population is exposed to MPs and that there are resultant health effects is sufficiently persuasive. Therefore, human exposure assessment and health effect studies of MPs should be carried out; however, there are no guidelines on how to assess the human exposure of MPs to date.

Currently, MPs are defined as plastic particles with a diameter of $5 \mathrm{~mm}$ or less $[1,2]$, although the physical properties and chemical compositions of MPs have not been standardized. Therefore, these non-standardized parameters can be variously defined according to the viewpoint of the research. This perspective supports recent efforts to develop reference materials for the standardized exposure assessment of MPs [31].

In future studies, it is considered necessary to estimate the total exposure to MPs by combining the evaluation results of individual exposure routes for the consideration of the characteristics of MPs based on the various exposure routes. In order to facilitate this estimation, a large number of individual path evaluation results with high reliability and validity should be collected. If the estimated exposure values evaluated in various exposure pathways are sufficiently established, the risk assessment of MPs based on the total exposure calculation might be possible. In addition, in order to discover biomarkers that can link human exposure and health effects, it needs to be necessarily considered to establish absorption, distribution, metabolism, and excretion pathways of MPs.

\section{Limitations}

In this study, a quality assessment of the selected articles was performed, but it was difficult to sufficiently judge each item of quality assessment. The exposure assessment method for MPs was not determined. Moreover, most of the studies were not actual human studies nor health effect assessment studies that evaluated clear health outcomes. These were studies that observed and reported primary phenomena. Therefore, the requisite evidence base could not be created. 


\section{Conclusions}

This systematic review identified articles on MPs exposure assessment that have been published to date, and mapped the viewpoints presented by classifying them into each stage of the pollutant-exposure-health outcome process. In addition, the research tools, such as analysis methods, risk factors, and biomarkers that were applied at each stage, were listed. Observations from this review that should be considered for future studies of MPs exposure assessment and health impact assessment include the followings:

1. MPs have various forms, chemical compositions, exposure routes, and delivery media. Therefore, their mechanisms of MP exposure to the human body and the patterns of their effects are diverse. Future studies of MP health effect assessment should be conducted by creating a scenario and identifying the expected types of exposure, and the results of these studies can be assimilated to suggest the total exposure estimates and integrated risk assessment of exposure to MPs.

2. There is a need for a standardized exposure assessment method that can quantify human exposure to MPs or a method that can predict MP exposure levels. Therefore, it is necessary to define MPs in terms of exposure evaluation and undertake studies to identify surrogates and markers of MP exposure. In PM studies, individual exposure can be calculated through PM concentrations of ambient air at the group level and the determination of indoor air quality at the individual level. However, to study MP exposure, more complicated evaluations and theoretical evidence to estimate exposure are necessary.

3. It is reasonable to assume that exposure to MPs has already occurred in the general population and that the health effects of the general population due to MPs exposure have already occurred. The risk of various chronic diseases may be increased by the toxicity of the chemical components of MPs. Furthermore, the identification of the chemical composition and the establishment of exposure assessment guidelines will facilitate the definition and listing of health outcomes caused by human exposure to MPs.

\section{Acknowledgement}

This study was supported by grants from the Environmental Health Center funded by the Korean Ministry of Environment.

\section{Conflict of interest}

The authors declare that they have no conflict of interest.

\section{CRediT author statement}

YMC: Review of articles, Conceptualization, Methodology, and Writing- Original draft preparation. KHC: Review of articles, Supervision, Writing- Reviewing and Editing.

\section{ORCID}

Yong Min Cho: 0000-0002-8999-8916

Kyung-Hwa Choi: 0000-0001-8206-4574.

\section{References}

[1] Thompson RC, Olsen Y, Mitchell RP, Davis A, Rowland SJ, John AW, et al. Lost at sea: where is all the plastic? Science 2004;304(5672):838.

[2] Collignon A, Hecq JH, Galgani F, Collard F, Goffart A. Annual variation in neustonic micro- and meso-plastic particles and zooplankton in the Bay of Calvi (Mediterranean-Corsica). Mar Pollut Bull 2014;79(1-2):293-298.

[3] Kershaw P, Rochman C. Sources, fate and effects of microplastics in the marine environment: part 2 of a global assessment. Reports and Studies-IMO/FAO/Unesco-IOC/WMO/IAEA/UN/UNEP Joint Group of Experts on the Scientific Aspects of Marine Environmental Protection (GESAMP) Eng No. 93. 2015

[4] Shen M, Zhang Y, Zhu Y, Song B, Zeng G, Hu D, et al. Recent advances in toxicological research of nanoplastics in the environment: A review. Environ Pollut 2019;252(Pt A):511-521.

[5] Moore CJ. Synthetic polymers in the marine environment: a rapidly increasing, long-term threat. Environ Res 2008;108(2):131-139.

[6] Li P, Wang X, Su M, Zou X, Duan L, Zhang H. Characteristics of plastic pollution in the environment: a review. Bull Environ Contam Toxicol 2020. 
[7] Wright SL, Kelly FJ. Plastic and human health: a micro issue? Environ Sci Technol 2017;51(12):6634-6647.

[8] Bouwmeester H, Hollman PC, Peters RJ. Potential health impact of environmentally released micro- and nanoplastics in the human food production chain: experiences from nanotoxicology. Environ Sci Technol 2015;49(15):8932-8947.

[9] Waring RH, Harris RM, Mitchell SC. Plastic contamination of the food chain: a threat to human health? Maturitas 2018;115:64-68.

[10] Woodruff TJ, Sutton P. The Navigation Guide systematic review methodology: a rigorous and transparent method for translating environmental health science into better health outcomes. Environ Health Perspect 2014;122(10):1007-1014.

[11] Moher D, Liberati A, Tetzlaff J, Altman DG, Group P. Preferred reporting items for systematic reviews and metaanalyses: the PRISMA statement. PLoS med 2009;6(7):e1000097.

[12] Kim SY, Park JE, Lee YJ, Seo HJ, Sheen SS, Hahn S, et al. Testing a tool for assessing the risk of bias for nonrandomized studies showed moderate reliability and promising validity. J Clin Rpidemiol 2013;66(4):408-414.

[13] Mishra S, charan Rath C, Das AP. Marine microfiber pollution: a review on present status and future challenges. Mar Pollut Bull 2019;140:188-197.

[14] Barboza LGA, Vethaak AD, Lavorante BR, Lundebye AK, Guilhermino L. Marine microplastic debris: An emerging issue for food security, food safety and human health. Mar Pollut Bull 2018;133:336-348.

[15] Bradney L, Wijesekara H, Palansooriya KN, Obadamudalige N, Bolan NS, Ok YS, et al. Particulate plastics as a vector for toxic trace-element uptake by aquatic and terrestrial organisms and human health risk. Environ Int 2019;131:104937.

[16] Toussaint B, Raffael B, Angers-Loustau A, Gilliland D, Kestens V, Petrillo M, et al. Review of micro- and nanoplastic contamination in the food chain. Food Addit Contam Part A Chem Anal Control Expo Risk Assess 2019;36(5):639-673.

[17] Welle F, Franz R. Microplastic in bottled natural mineral water - literature review and considerations on exposure and risk assessment. Food Addit Contam Part A Chem Anal Control Expo Risk Assess 2018;35(12):2482-2492.

[18] Schirinzi GF, Pérez-Pomeda I, Sanchís J, Rossini C, Farré M, Barceló D. Cytotoxic effects of commonly used nanomaterials and microplastics on cerebral and epithelial human cells. Environ Res 2017;159:579-587.

[19] Abbasi S, Keshavarzi B, Moore F, Turner A, Kelly FJ, Dominguez AO, et al. Distribution and potential health impacts of microplastics and microrubbers in air and street dusts from Asaluyeh County, Iran. Environ Pollut 2019;244:153-164.

[20] Vianello A, Jensen RL, Liu L, Vollertsen J. Simulating human exposure to indoor airborne microplastics using a Breathing Thermal Manikin. Sci Rep 2019;9(1):8670.

[21] Liu K, Wang X, Wei N, Song Z, Li D. Accurate quantification and transport estimation of suspended atmospheric microplastics in megacities: Implications for human health. Environ Int 2019;132:105127.

[22] Schwabl P, Köppel S, Königshofer P, Bucsics T, Trauner M, Reiberger T, et al. Detection of various microplastics in human stool: a prospective case series. Ann Intern Med 2019;171(7):453-457.

[23] Van Cauwenberghe L, Janssen CR. Microplastics in bivalves cultured for human consumption. Environ Pollut 2014;193:65-70.

[24] Fang C, Zheng R, Chen H, Hong F, Lin L, Lin H, et al. Comparison of microplastic contamination in fish and bivalves from two major cities in Fujian province, China and the implications for human health. Aquaculture 2019;512:734322.

[25] Winkler A, Santo N, Ortenzi MA, Bolzoni E, Bacchetta R, Tremolada P. Does mechanical stress cause microplastic release from plastic water bottles? Water Res 2019;166:115082.

[26] Luo T, Zhang Y, Wang C, Wang X, Zhou J, Shen M, et al. Maternal exposure to different sizes of polystyrene microplastics during gestation causes metabolic disorders in their offspring. Environ Pollut 2019;255(Pt 1):113122.

[27] Liao YL, Yang JY. Microplastic serves as a potential vector for $\mathrm{Cr}$ in an in-vitro human digestive model. Sci Total Environ 2020;703:134805.

[28] Stock V, Fahrenson C, Thuenemann A, Dönmez MH, Voss L, Böhmert L, et al. Impact of artificial digestion on the sizes and shapes of microplastic particles. Food Chem Toxicol 2020;135:111010. 
[29] Liu C, Li J, Zhang Y, Wang L, Deng J, Gao Y, et al. Widespread distribution of PET and PC microplastics in dust in urban China and their estimated human exposure. Environ Int 2019;128:116-124.

[30] Hwang J, Choi D, Han S, Choi J, Hong J. An assessment of the toxicity of polypropylene microplastics in human derived cells. Sci Total Environ 2019;684:657-669.

[31] Gerdes Z, Hermann M, Ogonowski M, Gorokhova E. A novel method for assessing microplastic effect in suspension through mixing test and reference materials. Sci Rep 2019;9(1):10695. 